\title{
Robust stability analysis for linear systems subjected to time-varying uncertainties within the framework of nest algebra
}

\author{
Xiaoping $\mathrm{Xu}^{\mathrm{a}, *}$, Liu Liu ${ }^{\mathrm{b}}$ \\ a School of Mathematical Sciences, Ocean University of China, Qingdao 266100, China \\ b School of Mathematical Sciences, Dalian University of Technology, Dalian 116024, China \\ *Corresponding author, e-mail: xxpouc@163.com
}

Received 12 Sep 2017

Accepted 29 Nov 2017

\begin{abstract}
In this paper, we consider the robust stabilization problem for linear systems subjected to time-varying uncertainties within the framework of nest algebra. For four types of plant perturbations, we obtain some new sufficient conditions or necessary and sufficient conditions for robust stabilization without using coprime factorization. We also give some examples to show the validity of our new results.
\end{abstract}

KEYWORDS: stabilization, linear time-varying system, perturbation

MSC2010: 93D99

\section{INTRODUCTION}

Stability is a basic and important concept in systems theory. It has been discussed in many frameworks $^{1-4}$. For linear time-varying (LTV) systems, robust stability $^{5}$, asymptotic stability ${ }^{6,7}$, and simultaneous stability ${ }^{8,9}$ have been studied. In particular, the robust stability of a standard feedback configuration with plant $L$ and compensator $C$ has been studied in several different frameworks ${ }^{1,2,4}$. It is concerned with the problem of designing a controller ${ }^{10,11}$ that simultaneously stabilizes all plants for some set. Robust stability has been widely used in engineering.

Since the development of $H^{\infty}$ control theory in the nineties, a lot of insights have been obtained by considering its time-varying analogue on an appropriate complex Hilbert space of input-output signals. In the context of operator theory, the set of stable and causal LTV systems is represented by a nest algebra ${ }^{4,12}$. The control theory for infinitedimensional LTV systems was developed based on the nest algebra approach. Currently, there have been many methods to study the closed loop stability of causal LTV systems in the framework of nest algebra. These methods are based on graph theory, coprime factorization theory, and gap metric theory ${ }^{4}$. Recently, some new results have been obtained in the framework of nest algebra: for example, the stability analysis in Refs. 13-16, and the simultaneous stabilization in Refs. 8, 9, 17, 18. Within the framework of nest algebra, the robust stability problem has been considered mainly in the coprime-factor perturbation case ${ }^{13,17,19,20}$, but the necessary and sufficient conditions have not been considered. Furthermore, the computation of the coprime factorization has not been well studied for a general LTV system in the framework of nest algebra. Hence, the design of new robust (simultaneous, internal, strong) stabilization criteria in the form of fewer coprime factorizations needs further study.

In the paper, we will use the definition of closed loop stability to discuss robust stability. We focus on four types of time-varying perturbations for LTV systems $^{2}$. Without using coprime factorization, we only consider the system $L$ and controller $C$ themselves. We obtain some sufficient conditions or necessary and sufficient conditions for robust stabilization. In particular, when the controller is stable, we obtain the necessary and sufficient conditions for robust stabilization. Furthermore, the controller designing problem and the simultaneous stabilization problem can be solved by using the necessary and sufficient conditions. Our method is clearly different to that of the robust stabilization using coprime-factor uncertainty ${ }^{17,19,21}$. The method is 
original and the results are new. To the best of our knowledge, there are no corresponding results in the literature for robust stability in the framework of nest algebra.

\section{PRELIMINARIES}

Let $\mathscr{H}$ be a complex infinite-dimensional sequence space with the standard Euclidean norm $\|x\|^{2}=\sum_{i=0}^{\infty}\left|x_{i}\right|^{2}<\infty$, where $x=\left(x_{0}, x_{1}, x_{2}, \ldots\right) \in$ $\mathscr{H}, x_{i} \in \mathrm{C}$. Clearly, $\mathscr{H}$ is a separable Hilbert space with the standard inner product $(x, y)=\sum_{i=0}^{\infty} x_{i} \bar{y}_{i}$, where $\bar{y}_{i}$ is the conjugation of $y_{i}$.

A linear operator $T: D(T) \subset \mathscr{H} \rightarrow \mathscr{H}$ is bounded with norm $\|T\|$ if its domain $D(T):=\{u \in \mathscr{H}: T u \in \mathscr{H}\}=\mathscr{H}$ and $\|T\|=\sup _{x \in D(T),\|x\| \leqslant 1}\|T x\|<\infty$. Let $\mathscr{B}(\mathscr{H})$ denote the set of all bounded linear operators on $\mathscr{H}$. For $T \in \mathscr{B}(\mathscr{H}), \operatorname{Ran} T$ denotes the range $\{T x: x \in \mathscr{H}\}$ of $T$ and KerT denotes the kernel $\{x \in \mathscr{H}: T x=0\}$ of $T$.

Let $\mathscr{H}_{e}=\left\{\left(x_{0}, x_{1}, x_{2}, \ldots\right): x_{i} \in \mathrm{C}\right\}$ denote the extended space of $\mathscr{H}$. For each $n \geqslant 0$, the standard truncation projection $P_{n}$ on $\mathscr{H}$ and $\mathscr{H}_{e}$ is defined by

$P_{n}\left(x_{0}, x_{1}, \ldots, x_{n}, x_{n+1}, \ldots\right)=\left(x_{0}, x_{1}, \ldots, x_{n}, 0,0, \ldots\right)$,

with $P_{-1}=0, P_{\infty}=I$. $P_{n}$ sets all outputs after time $n$ to zero, so the projection sequence $\left\{P_{n}\right\}_{n=-1}^{\infty}$ is crucial to the physical notion of causality for linear systems.

A linear transformation $L$ on $\mathscr{H}_{e}$ is causal if $P_{n} L=P_{n} L P_{n}$ for all $-1 \leqslant n \leqslant \infty$. A linear system on $\mathscr{H}_{e}$ is a causal linear transformation on $\mathscr{H}_{e}$, which is continuous with respect to the resolution topology. A linear system $L$ is stable if its restriction to $\mathscr{H}$ is a bounded operator ${ }^{4}$.

Clearly, the set of linear systems on $\mathscr{H}_{e}$ is an algebra with respect to standard addition and multiplication. We denote this algebra by $\mathscr{L}$. It is easy to check that any element in $\mathscr{L}$ is an infinitedimensional lower triangular matrix with respect to the standard basis. The set of stable linear systems on $\mathscr{H}_{e}$, denoted by $\mathscr{S}$, is a weakly closed algebra containing the identity. $\mathscr{S}$ is a nest algebra in the operator theory literature. An important subalgebra of $\mathscr{L}$ is the algebra $\mathscr{T}$ of time-invariant linear systems. This algebra consists of the lower matrices that are Toeplitz (although not necessarily bounded operators). We have $\mathscr{T} \cap \mathscr{S}=H^{\infty}$.

A nest is a chain $\mathscr{N}$ of closed subspaces of a Hilbert space $\mathscr{H}$ containing $\{0\}$ and $\mathscr{H}$, which is closed under intersection and closed span. The nest algebra determined by $\mathscr{N}$ is

$$
\mathscr{T}(\mathscr{N})=\{T \in \mathscr{B}(\mathscr{H}): T N \subseteq N, \quad \forall N \in \mathscr{N}\} .
$$

In the following, we consider the LTV systems in discrete time. The standard way of describing such a system is by using the time-varying difference equations

$$
\begin{aligned}
x_{k+1} & =a_{k} x_{k}+b_{k} u_{k}, \\
x_{0} & =0, \\
y_{k} & =d_{k} x_{k}+e_{k} u_{k},
\end{aligned}
$$

where $u=\left(u_{0}, u_{1}, u_{2}, \ldots\right)^{T}, x=\left(x_{0}, x_{1}, x_{2}, \ldots\right)^{T}$, $y=\left(y_{0}, y_{1}, y_{2}, \ldots\right)^{T} \in \mathscr{H}_{e}$, and $\left\{a_{k}\right\},\left\{b_{k}\right\},\left\{d_{k}\right\}$, $\left\{e_{k}\right\}$ are sequences of matrices of appropriate dimensions. Hence the above system $L$ can be easily computed from the equations and is given by

$$
L=\left[\begin{array}{ccccc}
e_{0} & & & & \\
d_{1} b_{0} & e_{1} & & & \\
d_{2} a_{1} b_{0} & d_{2} b_{1} & e_{2} & & \\
d_{3} a_{2} a_{1} b_{0} & d_{3} a_{2} b_{1} & d_{3} b_{2} & e_{3} & \\
\vdots & \vdots & \vdots & \ldots & \ddots
\end{array}\right] .
$$

Clearly, the system $L$ is an infinite-dimensional lower triangular matrix.

For $L, C \in \mathscr{L}$, we consider the standard feedback configuration (Fig. 1) with plant $L$ and compensator $C$, where the closed loop system equation is

$$
\left[\begin{array}{l}
u_{1} \\
u_{2}
\end{array}\right]\left[\begin{array}{cc}
I & C \\
L & -I
\end{array}\right]\left[\begin{array}{l}
e_{1} \\
e_{2}
\end{array}\right] .
$$

The system $\{L, C\}$ is well posed if

$$
\left[\begin{array}{cc}
I & C \\
L & -I
\end{array}\right]
$$

is invertible. Its inverse is given by the transfer matrix

$$
H(L, C)=\left[\begin{array}{cc}
(I+C L)^{-1} & C(I+L C)^{-1} \\
L(I+C L)^{-1} & -(I+L C)^{-1}
\end{array}\right] .
$$

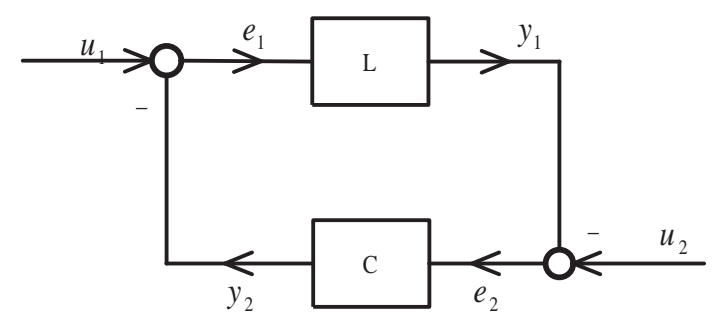

Fig. 1 The standard feedback configuration. 
Let $\mathscr{D}(L)=\{u \in \mathscr{H}: L u \in \mathscr{H}\}$ and $\mathscr{D}(C)=\{u \in \mathscr{H}$ : $\mathrm{Cu} \in \mathscr{H}\}$. Then

$$
\left[\begin{array}{cc}
I & C \\
L & -I
\end{array}\right]
$$

can be regarded as a linear transformation from $\mathscr{D}(L) \oplus \mathscr{D}(C)$ into $\mathscr{H} \oplus \mathscr{H}$.

Definition 1 [Ref. 4] The closed loop system $\{L, C\}$ is stable if all the entries of $H(L, C)$ are stable systems on $\mathscr{H}$. The plant $L$ is stabilizable if there exists $C \in \mathscr{L}$ such that the closed loop system $\{L, C\}$ is stable. The plant $L$ is strongly stabilizable if $C \in \mathscr{S}$.

The notion of robustness can be described as follows.

Definition 2 [Ref. 2] Suppose that the system $L$ belongs to a set $\mathscr{P}$. A controller $C$ provides robust stability if it provides internal stability for every plant in $\mathscr{P}$.

In this paper, we mainly focus on the following four types of plant perturbations:

$$
\begin{aligned}
A\left(L_{0}\right) & =\left\{L: L=L_{0}+\Delta\right\} ; \\
M\left(L_{0}\right) & =\left\{L: L=(I+\Delta) L_{0}\right\} ; \\
D_{1}\left(L_{0}\right) & =\left\{L: L=L_{0}\left(I+\Delta L_{0}\right)^{-1}\right\} ; \\
D_{2}\left(L_{0}\right) & =\left\{L: L=L_{0}(I+\Delta)^{-1}\right\},
\end{aligned}
$$

where $L_{0}$ is a given system and $\Delta$ is some perturbation set.

Next, we state some plant perturbations when $\Delta \in \mathscr{S}$. Suppose $r$ is a constant, the class of additive perturbations of $L_{0}$ is denoted by $A\left(L_{0}, r\right)$ :

$$
A\left(L_{0}, r\right)=\left\{L: L=L_{0}+\Delta,\|\Delta\|<r\right\} .
$$

Similarly, the class of the other perturbations of $L_{0}$ is denoted by

$$
\begin{aligned}
& M\left(L_{0}, r\right)=\left\{L: L=(I+\Delta) L_{0},\|\Delta\|<r\right\} ; \\
& D_{1}\left(L_{0}, r\right)=\left\{L: L=L_{0}\left(I+\Delta L_{0}\right)^{-1},\|\Delta\|<r\right\} ; \\
& D_{2}\left(L_{0}, r\right)=\left\{L: L=L_{0}(I+\Delta)^{-1},\|\Delta\|<r\right\} .
\end{aligned}
$$

In particular, for an additive perturbation, when $L_{0}$ has a right coprime factorization $N_{0} D_{0}^{-1}$, it is reduced to the coprime-factor perturbation. Let $S\left(N_{0}, D_{0}, r\right)$ denote the class of coprime-factor perturbations. It consists of all plants $L$ satisfying $L=$ $N D^{-1}$, where

$$
\left\|\left[\begin{array}{l}
N-N_{0} \\
D-D_{0}
\end{array}\right]\right\|<r
$$

\section{SUFFICIENCY OF ROBUST STABILIZATION}

In this section, for the plant with different perturbations, we give some sufficient conditions for their stability.

Theorem 1 Suppose $\left\{L_{0}, C\right\}$ is stable, $\Delta \in \mathscr{S}$, and $\|\Delta\|<r$. Then for the plant with a different perturbation, we have the following results.

(i) If

$$
\left\|C\left(I+L_{0} C\right)^{-1}\right\| \leqslant \frac{1}{r},
$$

then $C$ stabilizes all $L$ in $A\left(L_{0}, r\right)$.

(ii) If $\left\|L_{0} C\left(I+L_{0} C\right)^{-1}\right\| \leqslant 1 / r$ then $C$ stabilizes all $L$ in $M\left(L_{0}, r\right)$.

(iii) If $\left\|L_{0}\left(I+C L_{0}\right)^{-1}\right\| \leqslant 1 / r$ then $C$ stabilizes all $L$ in $D_{1}\left(L_{0}, r\right)$.

(iv) If $\left\|\left(I+C L_{0}\right)^{-1}\right\| \leqslant 1 / r$ then $C$ stabilizes all $L$ in $D_{2}\left(L_{0}, r\right)$.

Proof: (i) Suppose $\left\|C\left(I+L_{0} C\right)^{-1}\right\| \leqslant 1 / r$, for arbitrary $L$ in $A\left(L_{0}, r\right)$. We have $\Delta=L-L_{0}$. Let $F=C\left(I+L_{0} C\right)^{-1}$. By (1) and the fact that $\|\Delta\|<r$, we have $\|\Delta\|\|F\|<1$. Then $I+\Delta F$ and $I+F \Delta$ are both invertible in $\mathscr{S}$. By the fact that

$$
\begin{aligned}
I+L C & =I+\left(\Delta+L_{0}\right) C \\
& =\left[I+\Delta C\left(I+L_{0} C\right)^{-1}\right]\left(I+L_{0} C\right) \\
& =(I+\Delta F)\left(I+L_{0} C\right),
\end{aligned}
$$

and $I+L_{0} C$ is invertible in $\mathscr{S}$ since $C$ stabilizes $L_{0}$, we obtain that $I+L C$ is invertible in $\mathscr{S}$. Let

$$
H(L, C)=\left[\begin{array}{ll}
H_{11} & H_{12} \\
H_{21} & H_{22}
\end{array}\right] .
$$

Then

$$
\begin{aligned}
H_{11}= & (I+L C)^{-1} \\
= & \left(I+L_{0} C\right)^{-1}(I+\Delta F)^{-1} \in \mathscr{S} ; \\
H_{12}= & C(I+L C)^{-1} \\
= & C\left(I+L_{0} C\right)^{-1}(I+\Delta F)^{-1} \in \mathscr{S} ; \\
H_{21}= & L(I+C L)^{-1} \\
= & \left(\Delta+L_{0}\right)\left(I+C L_{0}\right)^{-1}\left[I+C \Delta\left(I+C L_{0}\right)^{-1}\right]^{-1} \\
= & \Delta\left(I+C L_{0}\right)^{-1}\left[I+C \Delta\left(I+C L_{0}\right)^{-1}\right]^{-1} \\
& +L_{0}\left(I+C L_{0}\right)^{-1}\left[I+C \Delta\left(I+C L_{0}\right)^{-1}\right]^{-1} .
\end{aligned}
$$

As $I+\Delta F=I+\Delta C\left(I+L_{0} C\right)^{-1}=I+\Delta\left(I+C L_{0}\right)^{-1} C$ is invertible in $\mathscr{S}$, and by the fact that $I+A B$ is invertible in $\mathscr{S}$ if and only if $I+B A$ is invertible in $\mathscr{S}$, we have that $I+C \Delta\left(I+C L_{0}\right)^{-1}$ is invertible in $\mathscr{S}$. 
Since $\Delta \in \mathscr{S},\left(I+C L_{0}\right)^{-1} \in \mathscr{S}$ and $L_{0}\left(I+C L_{0}\right)^{-1} \in$ $\mathscr{S}$, we have $H_{21} \in \mathscr{S}$ and

$$
H_{22}=(I+C L)^{-1}=(I+F \Delta)^{-1}\left(I+C L_{0}\right)^{-1} \in \mathscr{S},
$$

then $C$ stabilizes all $L$ in $A\left(L_{0}, r\right)$.

(ii) For $L$ in $M\left(L_{0}, r\right)$, we have $L=(I+\Delta) L_{0}$, where $\Delta \in \mathscr{S}$ and $\|\Delta\|<r$. Let $G=L_{0} C\left(I+L_{0} C\right)^{-1}$. We have $\|G\| \leqslant 1 / r$. By the small gain theorem, we have that $I+\Delta G$ is invertible. Since

$$
\begin{aligned}
I+L C & =I+(I+\Delta) L_{0} C \\
& =\left[I+\Delta L_{0} C\left(I+L_{0} C\right)^{-1}\right]\left(I+L_{0} C\right) \\
& =(I+\Delta G)\left(I+L_{0} C\right),
\end{aligned}
$$

we obtain that $I+L C$ is invertible in $\mathscr{S}$. The rest of the proof is similar to that of (i).

The proofs of (iii) and (iv) are similar to that of

Remark 1 We remark that all the results are obtained without using any coprime factorization of the system $L_{0}$ and controller $C$. Currently, since it is still open how to compute the coprime factorization of a general LTV system in the framework of nest algebra, the design of new robust (simultaneous, internal, strong) stabilization criteria in the form of fewer coprime factorizations is one of the hot research subjects in robust control theory in the framework of nest algebra. Hence our work has some contributions from this point of view.

Next we give an example to explain the efficiency of Theorem 1(i).

Example 1 Take $r=\frac{4}{3}$ and consider the following systems:

$$
\begin{aligned}
L_{0} & =\left[\begin{array}{cccccc}
1 & & & & & \\
2 & 2 & & & & \\
3 & 3 & 3 & & & \\
\vdots & \vdots & \vdots & \ddots & & \\
n & n & n & \cdots & n & \\
\vdots & \vdots & \vdots & \cdots & \cdots & \ddots
\end{array}\right], \\
C & =\left[\begin{array}{ccccccc}
1 & & & & & & \\
-1 & \frac{1}{2} & & & & & \\
0 & -\frac{1}{2} & \frac{1}{3} & & & & \\
0 & 0 & -\frac{1}{3} & \frac{1}{4} & & & \\
\vdots & \vdots & \cdots & \ddots & \ddots & & \\
0 & 0 & \cdots & \cdots & -\frac{1}{n-1} & \frac{1}{n} & \\
\vdots & \vdots & \cdots & \cdots & \cdots & \ddots & \ddots
\end{array}\right] .
\end{aligned}
$$

Clearly $C$ stabilizes $L_{0}$. Note that

$$
\begin{aligned}
& C\left(I+L_{0} C\right)^{-1} \\
& \quad=\left[\begin{array}{ccccccc}
\frac{1}{2} & & & & & & \\
-\frac{1}{2} & \frac{1}{4} & & & & & \\
0 & -\frac{1}{4} & \frac{1}{6} & & & & \\
0 & 0 & -\frac{1}{6} & \frac{1}{8} & & & \\
\vdots & \vdots & \ldots & \ddots & \ddots & & \\
0 & 0 & 0 & \cdots & -\frac{1}{2(n-1)} & \frac{1}{2 n} & \\
\vdots & \vdots & \vdots & \cdots & \ldots & \ddots & \ddots
\end{array}\right] .
\end{aligned}
$$

It is obvious that $\left\|C\left(I+L_{0} C\right)^{-1}\right\|=\frac{1}{2}<1 / r$. For $\Delta \in \mathscr{S}$, take $\|\Delta\|=1<r$. Choose

$$
\Delta=\left[\begin{array}{ccccc}
1 & & & & \\
\delta_{21} & 1 & & & \\
\delta_{31} & \delta_{32} & 1 & & \\
\delta_{41} & \delta_{42} & \delta_{43} & 1 & \\
\vdots & \vdots & \vdots & \vdots & \ddots
\end{array}\right]
$$

where $\left|\delta_{i j}\right|<1, i>j$. Using Theorem 1(i), we obtain that $C$ stabilizes $L_{0}+\Delta$.

In the end of the section, we give an example to explain that the condition $\left\|C\left(I+L_{0} C\right)^{-1}\right\| \leqslant 1 / r$ is not necessary for $C$ to stabilize all $L$ in $A\left(L_{0}, r\right)$ in Theorem 1(i).

Example 2 Take $r=\frac{1}{3}$. We consider the following systems:

$$
\begin{aligned}
L_{0} & =\left[\begin{array}{cccccc}
\frac{1}{4} & 0 & 0 & 0 & \ldots & \ldots \\
\frac{1}{4} & \frac{1}{4} & 0 & 0 & \ldots & \ldots \\
\frac{1}{8 \cdot 3^{2}} & 0 & \frac{1}{4} & 0 & \ldots & \ldots \\
\vdots & \vdots & \vdots & \ddots & \ldots & \ldots \\
\frac{1}{8 \cdot n^{2}} & 0 & 0 & \ldots & \frac{1}{4} & \ldots \\
\vdots & \vdots & \vdots & \ldots & \ldots & \ddots
\end{array}\right], \\
C & =\left[\begin{array}{ccccc}
0 & 0 & 0 & 0 & \ldots \\
4 & 0 & 0 & 0 & \ldots \\
\frac{2}{3^{2}} & 0 & 0 & 0 & \ldots \\
\frac{2}{4^{2}} & 0 & 0 & 0 & \ldots \\
\vdots & \vdots & \vdots & \vdots & \ldots \\
\frac{2}{n^{2}} & 0 & 0 & 0 & \ldots \\
\vdots & \vdots & \vdots & \vdots & \ddots
\end{array}\right] .
\end{aligned}
$$

Clearly $C$ stabilizes $L_{0}$. For $\Delta \in \mathscr{S}$, take $\|\Delta\|=\frac{1}{5}<r$ 
and choose

$$
\Delta=\left[\begin{array}{ccccc}
\frac{1}{5} & & & & \\
\delta_{21} & \frac{1}{5} & & & \\
\delta_{31} & \delta_{32} & \frac{1}{5} & & \\
\delta_{41} & \delta_{42} & \delta_{43} & \frac{1}{5} & \\
\vdots & \vdots & \vdots & \vdots & \ddots
\end{array}\right]
$$

where $\left|\delta_{i j}\right|<\frac{1}{5}, i>j$. Clearly $C$ also stabilizes $L_{0}+$ $\Delta$. However, we have

$$
C\left(I+L_{0} C\right)^{-1}=\left[\begin{array}{ccccc}
0 & 0 & 0 & 0 & \cdots \\
4 & 0 & 0 & 0 & \cdots \\
\frac{2}{3^{2}} & 0 & 0 & 0 & \cdots \\
\frac{2}{4^{2}} & 0 & 0 & 0 & \cdots \\
\vdots & \vdots & \vdots & \vdots & \cdots \\
\frac{2}{n^{2}} & 0 & 0 & 0 & \cdots \\
\vdots & \vdots & \vdots & \vdots & \ddots
\end{array}\right]
$$

and $\left\|C\left(I+L_{0} C\right)^{-1}\right\|=4>1 / r$.

\section{STRONGLY ROBUST STABILIZATION}

Lemma 1 Suppose $C \in \mathscr{S}$. Then $\{L, C\}$ is stable if and only if $L(I+C L)^{-1} \in \mathscr{S}$.

Proof: Only if: it can be obtained by the stability definition of $\{L, C\}$. If: suppose $L(I+C L)^{-1} \in \mathscr{S}$, and let

$$
H(L, C)=\left[\begin{array}{ll}
H_{11} & H_{12} \\
H_{21} & H_{22}
\end{array}\right]
$$

Then

$$
\begin{aligned}
H_{21} & =L(I+C L)^{-1} \in \mathscr{S}, \\
H_{11} & =(I+C L)^{-1}=I-C(I+L C)^{-1} L \\
& =I-C L(I+C L)^{-1}=I-C H_{21} \in \mathscr{S}, \\
H_{12} & =C(I+L C)^{-1}=(I+C L)^{-1} C=H_{11} C \in \mathscr{S}, \\
H_{22} & =-(I+L C)^{-1}=L(I+C L)^{-1} C-I \\
& =H_{21} C-I \in \mathscr{S} .
\end{aligned}
$$

Hence $H(L, C) \in M(\mathscr{S})$, that is, $\{L, C\}$ is stable. In Lemma 1 , a necessary and sufficient condition for the strong stabilization is given. When the plant $L$ has the right coprime factorization $N M^{-1}$, Lemma 1 reduces to Theorem 6.6.3 in Ref. 4. Similarly, we have the following result when the plant is stable.

Lemma 2 Suppose $L \in \mathscr{S}$. Then $\{L, C\}$ is stable if and only if $C(I+L C)^{-1} \in \mathscr{S}$.

Next, we focus on the strongly robust stabilization of four different plant perturbations in Theorem 2, which is the main conclusion in the paper.
Theorem 2 Suppose $C \in \mathscr{S}$ stabilizes $L_{0}$, and $\Delta \in$ $\mathscr{S}$. We have the following results.

(i) C stabilizes $L \in A\left(L_{0}\right)$ if and only if $I+\Delta(I+$ $\left.C L_{0}\right)^{-1} C$ is invertible in $\mathscr{S}$.

(ii) C stabilizes $L \in M\left(L_{0}\right)$ if and only if $I+\Delta L_{0}(I+$ $\left.C L_{0}\right)^{-1} C$ is invertible in $\mathscr{S}$.

(iii) C stabilizes $L \in D_{1}\left(L_{0}\right)$ if and only if $I+\Delta L_{0}(I+$ $\left.C L_{0}\right)^{-1}$ is invertible in $\mathscr{S}$.

(iv) C stabilizes $L \in D_{2}\left(L_{0}\right)$ if and only if $I+\Delta(I+$ $\left.C L_{0}\right)^{-1}$ is invertible in $\mathscr{S}$.

Proof: (i) As $I+\Delta\left(I+C L_{0}\right)^{-1} C$ is invertible if and only if $I+C \Delta\left(I+C L_{0}\right)^{-1}$ is invertible, we only need to prove that $I+C \Delta\left(I+C L_{0}\right)^{-1}$ is invertible in $\mathscr{S}$. Let $T=I+C \Delta\left(I+C L_{0}\right)^{-1}$. Then

$$
\begin{aligned}
T & =\left(I+C L_{0}+C \Delta\right)\left(I+C L_{0}\right)^{-1} \\
& =\left[I+C\left(L_{0}+\Delta\right)\right]\left(I+C L_{0}\right)^{-1} .
\end{aligned}
$$

By Lemma 1, the controller $C$ stabilizes $L_{0}+\Delta$ if and only if $\left(L_{0}+\Delta\right)\left[I+C\left(L_{0}+\Delta\right)\right]^{-1} \in \mathscr{S}$, that is, $\left(L_{0}+\Delta\right)\left(I+C L_{0}\right)^{-1}\left[I+C \Delta\left(I+C L_{0}\right)^{-1}\right]^{-1} \in \mathscr{S}$. Only if: suppose $C$ stabilizes $L_{0}+\Delta$. Then $H_{11}=[I+$ $\left.C\left(L_{0}+\Delta\right)\right]^{-1} \in \mathscr{S}$. By the fact that

$$
\begin{aligned}
& H_{21}=\left(L_{0}+\Delta\right)\left[I+C\left(L_{0}+\Delta\right)\right]^{-1} \\
& \quad=L_{0}\left[I+C\left(L_{0}+\Delta\right)\right]^{-1}+\Delta\left[I+C\left(L_{0}+\Delta\right)\right]^{-1} \in \mathscr{S},
\end{aligned}
$$

and $\Delta \in \mathscr{S},\left[I+C\left(L_{0}+\Delta\right)\right]^{-1} \in \mathscr{S}$, we have $L_{0}[I+$ $\left.C\left(L_{0}+\Delta\right)\right]^{-1} \in \mathscr{S}$. By using (2), we obtain that $T$ is invertible in $\mathscr{S}$, and its inverse is given by $T^{-1}=$ $\left(I+C L_{0}\right)\left[I+C\left(L_{0}+\Delta\right)\right]^{-1} \in \mathscr{S}$.

If: suppose $T$ is invertible in $\mathscr{S}$, that is, $I+$ $C \Delta\left(I+C L_{0}\right)^{-1}$ is invertible in $\mathscr{S}$. We have

$$
\begin{aligned}
& \left(L_{0}+\Delta\right)\left[I+C\left(L_{0}+\Delta\right)\right]^{-1} \\
& =L_{0}\left(I+C L_{0}\right)^{-1}\left[I+C \Delta\left(I+C L_{0}\right)^{-1}\right]^{-1} \\
& \quad+\Delta\left(I+C L_{0}\right)^{-1}\left[I+C \Delta\left(I+C L_{0}\right)^{-1}\right]^{-1} \\
& \quad=L_{0}\left(I+C L_{0}\right)^{-1} T^{-1}+\Delta\left(I+C L_{0}\right)^{-1} T^{-1} \in \mathscr{S} .
\end{aligned}
$$

Hence $C$ stabilizes all $L$ in $A\left(L_{0}\right)$.

(ii) The result can be obtained by replacing $\Delta$ in (i) with $\Delta L_{0}$.

(iii) Let $G=I+\Delta L_{0}\left(I+C L_{0}\right)^{-1}$. Then

$$
\begin{aligned}
G & =\left(I+C L_{0}+\Delta L_{0}\right)\left(I+C L_{0}\right)^{-1} \\
& =\left[I+(C+\Delta) L_{0}\right]\left(I+C L_{0}\right)^{-1} .
\end{aligned}
$$

By Lemma 1 , the controller $C$ stabilizes $L_{0}(I+$ $\left.\Delta L_{0}\right)^{-1}$ if and only if $L_{0}\left(I+\Delta L_{0}\right)^{-1}\left[I+C L_{0}(I+\right.$ $\left.\left.\Delta L_{0}\right)^{-1}\right]^{-1} \in \mathscr{S}$, that is, $L_{0}\left[I+(C+\Delta) L_{0}\right]^{-1} \in \mathscr{S}$. Only if: suppose $C$ stabilizes $L_{0}\left(I+\Delta L_{0}\right)^{-1}$. Then 
$H_{11}=\left[I+C L_{0}\left(I+\Delta L_{0}\right)^{-1}\right]^{-1} \in \mathscr{S}$. By the fact that

$$
\begin{aligned}
H_{11} & =\left\{\left[\left(I+\Delta L_{0}\right)+C L_{0}\right]\left(I+\Delta L_{0}\right)^{-1}\right\}^{-1} \\
& =\left(I+\Delta L_{0}\right)\left[I+(C+\Delta) L_{0}\right]^{-1} \\
& =\left[I+(C+\Delta) L_{0}\right]^{-1}+\Delta L_{0}\left[I+(C+\Delta) L_{0}\right]^{-1},
\end{aligned}
$$

$\Delta \in \mathscr{S}$, and $L_{0}\left[I+(C+\Delta) L_{0}\right]^{-1} \in \mathscr{S}$, we have $[I+$ $\left.(C+\Delta) L_{0}\right]^{-1} \in \mathscr{S}$. By using (3), we obtain that $G$ is invertible in $\mathscr{S}$, and its inverse is given by $G^{-1}=$ $\left(I+C L_{0}\right)\left[I+(C+\Delta) L_{0}\right]^{-1} \in \mathscr{S}$.

If: suppose $G$ is invertible in $\mathscr{S}$. By (3), we know that $I+(C+\Delta) L_{0}$ is invertible in $\mathscr{S}$. Note that

$$
\begin{aligned}
L_{0} & {\left[I+(C+\Delta) L_{0}\right]^{-1} } \\
& =L_{0}\left[\left(I+C L_{0}\right)+\Delta L_{0}\right]^{-1} \\
& =L_{0}\left(I+C L_{0}\right)^{-1}\left[I+\Delta L_{0}\left(I+C L_{0}\right)^{-1}\right]^{-1} \\
& =L_{0}\left(I+C L_{0}\right)^{-1} G^{-1} \in \mathscr{S} .
\end{aligned}
$$

Hence $C$ stabilizes all $L$ in $D_{1}\left(L_{0}\right)$.

(iv) By Lemma 1 , the controller $C$ stabilizes $L_{0}(I+\Delta)^{-1}$ if and only if $L_{0}(I+\Delta)^{-1}\left[I+C L_{0}(I+\right.$ $\left.\Delta)^{-1}\right]^{-1} \in \mathscr{S}$. Since

$$
\begin{aligned}
& L_{0}(I+\Delta)^{-1}\left[I+C L_{0}(I+\Delta)^{-1}\right]^{-1} \\
& \quad=L_{0}\left(I+C L_{0}+\Delta\right)^{-1} \\
& \quad=L_{0}\left(I+C L_{0}\right)^{-1}\left[I+\Delta\left(I+C L_{0}\right)^{-1}\right]^{-1},
\end{aligned}
$$

we have $L_{0}\left(I+C L_{0}+\Delta\right)^{-1} \in \mathscr{S}$ and $L_{0}\left(I+C L_{0}\right)^{-1}[I+$ $\left.\Delta\left(I+C L_{0}\right)^{-1}\right]^{-1} \in \mathscr{S}$. If: let $R=I+\Delta\left(I+C L_{0}\right)^{-1}$. Then $R^{-1} \in \mathscr{S}$. Since $C \in \mathscr{S}$ stabilizes $L_{0}$, we have $L_{0}\left(I+C L_{0}\right)^{-1} \in \mathscr{S}$. Thus

$$
\begin{aligned}
& L_{0}(I+\Delta)^{-1}\left[I+C L_{0}(I+\Delta)^{-1}\right]^{-1} \\
& \quad=L_{0}\left(I+C L_{0}\right)^{-1}\left[I+\Delta\left(I+C L_{0}\right)^{-1}\right]^{-1} \\
& \quad=L_{0}\left(I+C L_{0}\right)^{-1} R^{-1} \in \mathscr{S} .
\end{aligned}
$$

Then $C$ stabilizes all $L$ in $D_{2}\left(L_{0}\right)$. Only if: suppose $C \in \mathscr{S}$ stabilizes $L_{0}(I+\Delta)^{-1}$. We have $U=[I+$ $\left.C L_{0}(I+\Delta)^{-1}\right]^{-1} \in \mathscr{S}$. Notice that

$$
U=(I+\Delta)\left(I+C L_{0}+\Delta\right)^{-1} .
$$

We have $\left(I+C L_{0}+\Delta\right)^{-1}=(I+\Delta)^{-1} U \in \mathscr{S}$. Then $R=I+\Delta\left(I+C L_{0}\right)^{-1}=\left(I+C L_{0}+\Delta\right)\left(I+C L_{0}\right)^{-1}$ is invertible and

$$
\begin{aligned}
R^{-1} & =\left[I+\Delta\left(I+C L_{0}\right)^{-1}\right]^{-1} \\
& =\left(I+C L_{0}\right)\left(I+C L_{0}+\Delta\right)^{-1} \\
& =\left(I+C L_{0}+\Delta\right)^{-1}+C L_{0}\left(I+C L_{0}+\Delta\right)^{-1} \in \mathscr{S} .
\end{aligned}
$$

Remark 2 In Theorem 2, the strongly robust stabilization is reduced to the invertibility of an operator. The results are also obtained without using any coprime factorization of the plant and the controller. Hence our method is effective as it is difficult or impossible to obtain a right coprime factorization in the time-varying case ${ }^{15}$. However, the results are invalid for $C \in \mathscr{L}$.

Remark 3 In Theorem 2, some necessary and sufficient conditions are obtained for strongly robust stabilization. In particular, the same controller $C \in$ $\mathscr{S}$ stabilizes both the plant $L_{0}$ and $L$. In some sense, it can be seen as a simultaneous stabilization problem. We do not need any factorization of the plant and controller in practice. So it is efficient and applicable.

Replacing the invertibility with the norm condition, we have the following corollary which is similar to Theorem 1.

Corollary 1 Suppose $C \in \mathscr{S}$ stabilizes $L_{0}$, and $\Delta \in$ $\mathscr{S}$.

(i) If $\|\Delta\|<1 /\left\|\left(I+C L_{0}\right)^{-1} C\right\|$, then $C$ stabilizes all $L$ in $A\left(L_{0}\right)$.

(ii) If $\|\Delta\|<1 /\left\|L_{0}\left(I+C L_{0}\right)^{-1} C\right\|$, then $C$ stabilizes all $L$ in $M\left(L_{0}\right)$.

(iii) If $\|\Delta\|<1 /\left\|L_{0}\left(I+C L_{0}\right)^{-1}\right\|$, then $C$ stabilizes all $L$ in $D_{1}\left(L_{0}\right)$.

(iv) If $\|\Delta\|<1 /\left\|\left(I+C L_{0}\right)^{-1}\right\|$, then $C$ stabilizes all $L$ in $D_{2}\left(L_{0}\right)$.

We now give two examples to demonstrate the validity of Theorem 2 .

Example 3 Consider the following systems:

$$
\begin{aligned}
L_{0} & =\left[\begin{array}{ccccc}
2 & & & & \\
-4 & 2 & & & \\
8 & -4 & 2 & & \\
-16 & 8 & -4 & 2 & \\
\vdots & \vdots & \vdots & \ddots & \ddots
\end{array}\right] \in \mathscr{L}, \\
\Delta & =\left[\begin{array}{cccc}
\delta_{11} & & & \\
\delta_{21} & \delta_{22} & & \\
\delta_{31} & \delta_{32} & \delta_{33} & \\
\vdots & \vdots & \ddots & \ddots
\end{array}\right] \in \mathscr{S} .
\end{aligned}
$$


Choose

$$
C=\left[\begin{array}{cccccc}
\frac{1}{4} & & & & & \\
-1 & \frac{1}{4} & & & & \\
0 & -1 & \frac{1}{4} & & & \\
\vdots & \vdots & \ddots & \ddots & & \\
0 & 0 & \ldots & -1 & \frac{1}{4} & \\
\vdots & \vdots & \ldots & \ldots & \ddots & \ddots
\end{array}\right] \in \mathscr{S} .
$$

Clearly, $C$ stabilizes $L_{0}$. We have

$$
\begin{aligned}
\left(I+C L_{0}\right)^{-1} & =\left[\begin{array}{cccccc}
\frac{2}{3} & & & & \\
\frac{4}{3} & \frac{2}{3} & & & \\
0 & \frac{4}{3} & \frac{2}{3} & & \\
0 & 0 & \frac{4}{3} & \frac{2}{3} & \\
\vdots & \vdots & \ldots & \ddots & \ddots
\end{array}\right], \\
\left(I+C L_{0}\right)^{-1} C & =\left[\begin{array}{cccccc}
\frac{1}{6} & & & & \\
-\frac{1}{3} & \frac{1}{6} & & & \\
-\frac{4}{3} & -\frac{1}{3} & \frac{1}{6} & & \\
0 & -\frac{4}{3} & -\frac{1}{3} & \frac{1}{6} & \\
\vdots & \vdots & \ddots & \ddots & \ddots
\end{array}\right]
\end{aligned}
$$

Clearly, $\left\|\left(I+C L_{0}\right)^{-1} C\right\|=\frac{4}{3}$. By Corollary 1(i), $C$ stabilizes all the systems of the form

$$
L_{0}+\Delta=\left[\begin{array}{cccc}
2+\delta_{11} & & & \\
-4+\delta_{21} & 2+\delta_{22} & & \\
8+\delta_{31} & -4+\delta_{32} & 2+\delta_{33} & \\
\vdots & \vdots & \ddots & \ddots
\end{array}\right]
$$

when $\|\Delta\|<1 /\left\|\left(I+C L_{0}\right)^{-1} C\right\|=\frac{3}{4}$. Since $I+\Delta(I+$ $\left.C L_{0}\right)^{-1} C$ equals

$$
\left[\begin{array}{cccc}
1+\frac{1}{6} \delta_{11} & & & \\
\frac{1}{6} \delta_{21}-\frac{1}{3} \delta_{22} & 1+\frac{1}{6} \delta_{22} & & \\
l_{31} & l_{32} & l_{33} & \\
\vdots & \vdots & \ldots & \ddots
\end{array}\right]
$$

where

$$
\begin{aligned}
& l_{31}=\frac{1}{6} \delta_{31}-\frac{1}{3} \delta_{32}-\frac{4}{3} \delta_{33}, \\
& l_{32}=\frac{1}{6} \delta_{32}-\frac{1}{3} \delta_{33}, \\
& l_{33}=1+\frac{1}{6} \delta_{33},
\end{aligned}
$$

we obtain that $C$ stabilizes all the systems of the form $L_{0}+\Delta$ if and only if $\delta_{n n} \neq-6(n=1,2, \ldots)$ by Theorem 2(i).

From Example 3, it is easy to find that Theorem 2(i) is more useful than Corollary 1(i). Actually,
Theorem 2 gives a deep and detailed description for $\Delta$.

We now give an example to demonstrate Theorem 2(iv).

Example 4 Consider the systems

$$
\begin{aligned}
& L_{0}=\left[\begin{array}{ccccccccc}
3 & & & & & & & \\
3 & 6 & & & & & & \\
3 & 6 & 9 & & & & & \\
3 & 6 & 0 & 12 & & & & \\
3 & 6 & 0 & 0 & 15 & & & \\
\vdots & \vdots & \vdots & \vdots & \ldots & \ddots & & \\
3 & 6 & 0 & 0 & \ldots & \cdots & 3 n & \\
\vdots & \vdots & \vdots & \vdots & \ldots & \ldots & \ldots & \ddots
\end{array}\right] \in \mathscr{L}, \\
& \Delta=\left[\begin{array}{ccccccc}
\delta_{11} & & & & & \\
\delta_{21} & \delta_{22} & & \\
\delta_{31} & \delta_{32} & \delta_{33} & \\
\vdots & \vdots & \ddots & \ddots
\end{array}\right] \in \mathscr{S}, \\
&
\end{aligned}
$$

where $\delta_{i i} \neq-1$ for $i=1,2, \ldots, n$. Choose

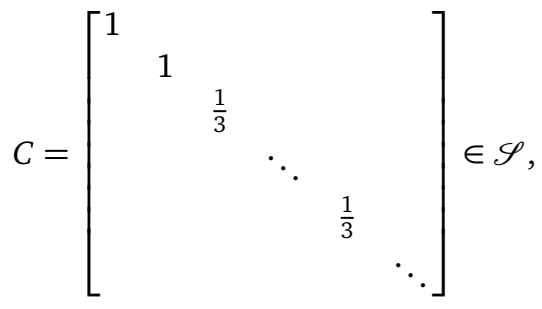

then $C$ stabilizes $L_{0}$, and we have

$$
\left(I+C L_{0}\right)^{-1}=\left[\begin{array}{ccccccc}
\frac{1}{4} & & & & & \\
-\frac{3}{28} & \frac{1}{7} & & & & \\
-\frac{1}{28 \times 4} & -\frac{1}{14} & \frac{1}{4} & & & \\
-\frac{1}{28 \times 5} & -\frac{2}{35} & 0 & \frac{1}{5} & & \\
-\frac{1}{28 \times 6} & -\frac{1}{21} & 0 & 0 & \frac{1}{6} & \\
\vdots & \vdots & \vdots & \ldots & \ldots & \ddots
\end{array}\right] .
$$

Clearly, $\left\|\left(I+C L_{0}\right)^{-1}\right\|=\frac{1}{4}$. By using Corollary 1 (iv), we obtain that $C$ stabilizes all the systems of the form $L_{0}(I+\Delta)^{-1}$ equal to

$$
\left[\begin{array}{cccccc}
\frac{3}{1+\delta_{11}} & & & & & \\
\frac{3}{1+\delta_{11}} & \frac{6}{1+\delta_{22}} & & & & \\
\frac{3}{1+\delta_{11}} & \frac{6}{1+\delta_{22}} & \frac{9}{1+\delta_{33}} & & & \\
\vdots & \vdots & \cdots & \ddots & & \\
\frac{3}{1+\delta_{11}} & \frac{6}{1+\delta_{22}} & 0 & \cdots & \frac{3 n}{1+\delta_{n n}} & \\
\vdots & \vdots & \vdots & \cdots & \cdots & \ddots
\end{array}\right]
$$


when $\|\Delta\|<1 /\left\|\left(I+C L_{0}\right)^{-1}\right\|=4$. Since $I+\Delta(I+$ $\left.C L_{0}\right)^{-1}$ equals

$$
\left[\begin{array}{ccccc}
1+\frac{1}{4} \delta_{11} & & & & \\
\frac{1}{4} \delta_{21}-\frac{3}{28} \delta_{22} & 1+\frac{1}{7} \delta_{22} & & & \\
a_{31} & a_{32} & a_{33} & & \\
a_{41} & a_{42} & a_{43} & a_{44} & \\
\vdots & \vdots & \vdots & \vdots & \ddots
\end{array}\right]
$$

where

$$
\begin{aligned}
& a_{31}=\frac{1}{4} \delta_{31}-\frac{3}{28} \delta_{32}-\frac{1}{112} \delta_{33}, \\
& a_{32}=\frac{1}{7} \delta_{32}-\frac{1}{14} \delta_{33}, \\
& a_{33}=1+\frac{1}{4} \delta_{33}, \\
& a_{41}=\frac{1}{4} \delta_{41}-\frac{3}{28} \delta_{42}-\frac{1}{112} \delta_{43}-\frac{1}{140} \delta_{44}, \\
& a_{42}=\frac{1}{7} \delta_{42}-\frac{1}{14} \delta_{43}-\frac{2}{35} \delta_{44}, \\
& a_{43}=\frac{1}{4} \delta_{43}, \\
& a_{44}=1+\frac{1}{5} \delta_{44},
\end{aligned}
$$

we obtain that $C$ stabilizes all the systems of the form $L_{0}(I+\Delta)^{-1}$ if and only if $\delta_{11} \neq-4, \delta_{22} \neq-7$, $\delta_{n n} \neq-(n+1)(n=3,4, \ldots)$ by Theorem 2(iv).

From Example 3 and Example 4, we see that the controller of a complicated system can be obtained by studying the controller $C$ of a simple system $L_{0}$. Thus the method is effective for designing the controller of plants having uncertainties.

\section{CONCLUSIONS}

In the paper, we mainly consider robust stability for LTV systems with different perturbations. Within the framework of nest algebra, by using the definition of closed loop stability, we obtain some sufficient conditions for robust stabilization. We also obtain some necessary and sufficient conditions for strongly robust stabilization. All the results are obtained without using any coprime factorization.

Acknowledgements: We would like to express our sincere thanks to the editors and the referees for their careful reading, valuable suggestions, timely review, and reply. This work was supported by the National Natural Science Foundation of China (Grant Nos. 11701537, 11501533).

\section{REFERENCES}

1. Vidyasagar M (1985) Control System Synthesis: A Factorization Approach, MIT Press, Cambridge.

2. Doyle J, Francis BA, Tannenbaum AR (1990) Feedback Control Theory, Macmillan, New York.

3. Briat C (2015) Linear Parameter-varying and Timedelay Systems-Analysis, Observation, Filtering and Control, Springer, Berlin.
4. Feintuch A (1998) Robust Control Theory in Hilbert Space, Springer, New York.

5. Djouadi SM, Charalambous CD (2010) Time-varying optimal disturbance minimization in presence of plant uncertainty. SIAM J Contr Optim 48, 3354-67.

6. Zhou B (2016) On asymptotic stability of linear timevarying systems. Automatica 68, 266-76.

7. Zhou B, Egorov AV (2016) Razumikhin and Krasovskii stability theorems for time-varying time-delay systems. Automatica 71, 281-91.

8. Yu TQ (2011) The transitivity in simultaneous stabilization. Syst Contr Lett 60, 1-6.

9. Yu TQ, Yan H (2011) Simultaneous controller design for time-varying linear systems. Syst Contr Lett 60, 1032-7.

10. Quadrat A (2004) On a general structure of the stabilizing controllers based on stable range. SIAM $J$ Contr Optim 42, 2264-85.

11. Sabau S, Martins NC (2014) Youla-like parametrizations subject to QI subspace constraints. IEEE Trans Automat Contr 59, 1411-22.

12. Davidson KR (1988) Nest Algebras, Pitman Research Notes in Mathematics Series, vol 191, Longman.

13. Liu L, Lu YF (2011) Stability analysis for time-varying systems via quadratic constraints. Syst Contr Lett $\mathbf{6 0}$, 832-9.

14. Liu L, Lu YF (2013) Necessary and sufficient conditions to the transitivity in simultaneous stabilisation of time-varying systems. IET Contr Theor Appl 7, 1834-42.

15. Liu L, Lu YF (2014) Stabilizability, representations and factorizations for time-varying linear systems. Syst Contr Lett 66, 58-64.

16. Lu YF, Gong $\mathrm{T}$ (2011) On stabilization for discrete linear time-varying systems. Syst Contr Lett 60, 1024-31.

17. Yu TQ, Wang CM (2012) Simultaneous robust stabilization problem of linear systems via transitivity approach. Advances in Computer, Communication, Control and Automation, vol 121, Springer, pp 353-60.

18. Yu TQ (2017) Simultaneously stabilizing controllers subject to QI subspace constraints. Eur J Contr 37, 63-9.

19. Lu YF, Xu XP (2008) The stabilization problems for discrete time-varying linear systems. Syst Contr Lett 57, 936-9.

20. Djouadi SM (2007) Robustness in the gap metric and coprime factor perturbations. IEEE Control and Decision Conference, New Orleans, pp 6400-5.

21. Feintuch A (1993) Robustness for time-varying systems. Math Contr Signal Syst 6, 247-63. 